\title{
The structure and evolution of global solar magnetic fields
}

\author{
Duncan H. Mackay \\ School of Mathematics and Statistics, University of St Andrews, St Andrews, Fife, KY16 8HB \\ email: duncan@mcs.st-and.ac.uk
}

\begin{abstract}
This review will discuss both observational and theoretical aspects of the Sun's global magnetic field. First recent observations will be described, along with the main physical processes leading to the time evolution and structure of the global field. Following this, recent theoretical models of both the global surface and coronal magnetic field will be presented. The application of these models to the structure of the corona, formation of solar filaments, the onset of CMEs and finally the origin and variation of the Sun's open flux will be discussed.
\end{abstract}

Keywords. Sun: activity, sun: corona, sun: magnetic fields

\section{Introduction}

In this review, our present day understanding of the Sun's global magnetic field is considered from both observational and theoretical aspects. To date three solar cycles worth of continuous data has been collected on the distribution and evolution of the Sun's photospheric magnetic field. An illustration of this can be seen in Figure 1 which shows the magnetic butterfly diagram (from Hathaway 2010). The plot illustrates key features in the distribution and evolution of the magnetic field, such as: emergence latitudes, 11 year polar field reversal, Joys' Law and Hales polarity law. As the global field evolves through this cyclic evolution it produces a wide range of phenomena in the corona such as solar filaments (Tandberg-Hanssen 1995; Mackay et al. 2010), variations in the Open Flux (Balogh et al. 1995; Lockwood et al. 1999) and eruptive phenomena such as Coronal Mass Ejections (Forbes et al. 2006; Cremades \& St. Cyr 2007). In the following we will describe models constructed to consider both the photospheric and coronal magnetic fields, along with the new generation of 3D MHD models.

\section{Magnetic flux transport models}

Magnetic flux transport simulations (Sheeley 2005) are designed to model the largescale, long time evolution of the radial magnetic field, $B_{r}$, at the solar photosphere. The four key physical effects included in these models are flux emergence, differential rotation, meridional flow and supergranular diffusion. Since the early flux transport models were produced (Wang et al. 1989) new variations have emerged to include additional physical effects (Schrijver 2001; Baumann et al. 2006). Applications have shown that flux transport models may accurately reproduce the essential characteristics of the evolution of magnetic fields not only on the Sun (Sheeley 2005) but also in other stars (Schrijver \& Title 2001), where in some cases different transport coefficients are required to match magnetic field observations (Mackay et al. 2004). Often, output from flux transport models are used as lower boundary conditions for coronal field modelling. Applications are now discussed. 


\section{PFSS models and corona null points}

To date, the most common technique for modelling the global coronal magnetic field of the Sun is through Potential Field Source Surface Models (PFSS, Schatten et al. 1969). Such models are simple to construct, as they require only the radial magnetic field at the solar photosphere to be specified (either from observations or flux transport models) and assume zero electric current in the corona. Recently Cook et al. (2009) used magnetic flux transport models combined with PFSS models to consider the origin and variation of coronal null points over two solar cycles. Null points are a key topological feature in the Magnetic Breakout Model of CMEs (Antiochos et al. 1999). Results show that the number of coronal null points vary in phase with the solar cycle, with more at cycle maximum than minimum. The majority of coronal nulls form above active latitudes and lie in the low corona (below $175,000 \mathrm{~km}$ ). This shows that the complex active latitude field is more important for the existence of the nulls compared to the overlying global dipole. While a significant number of nulls (15-17) are present each day, due to their low height only $50 \%$ have more flux lying below the null than above. This is a necessary condition for breakout to occur. Therefore, while the Magnetic Breakout Model may be an important mechanism for CMEs, other mechanisms must also act to account for the observed numbers (Cremades \& St. Cyr 2007; Barnes 2007). While potential field models are useful as a first approximation to the coronal field, it is known that the corona is non-potential due to eruptive phenomena that are present. Global non-potential models are now discussed. First, long-term reduced MHD models are considered, then steady state full MHD models.

\section{Global, long term non-potential models}

Recently van Ballegooijen et al. (2000) and Mackay \& van Ballegooijen (2006) have developed a new technique to study the global, long-term evolution of coronal magnetic fields. The technique follows the build-up of free magnetic energy and electric currents in the corona. Such effects are required in order to explain many eruptive phenomena found on the Sun. The technique has two components. The first component, which represents the solar photosphere, is a data driven magnetic flux transport model. This reproduces a continuous time evolution of the observed magnetic flux seen on the Sun over periods of

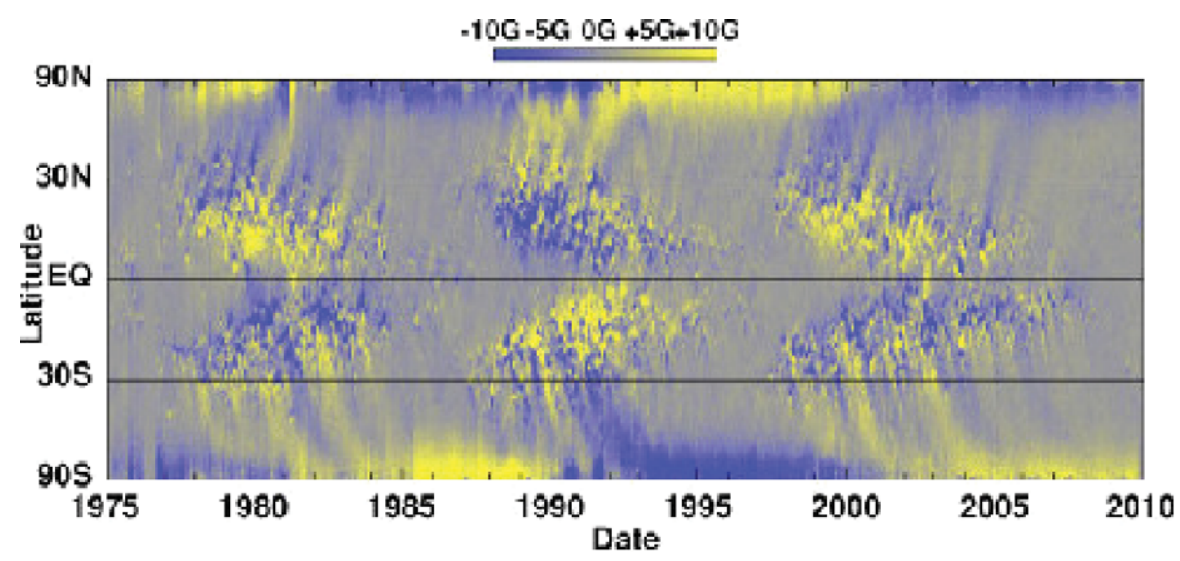

Figure 1. Magnetic butterfly Diagram (from Hathaway (2010)) 


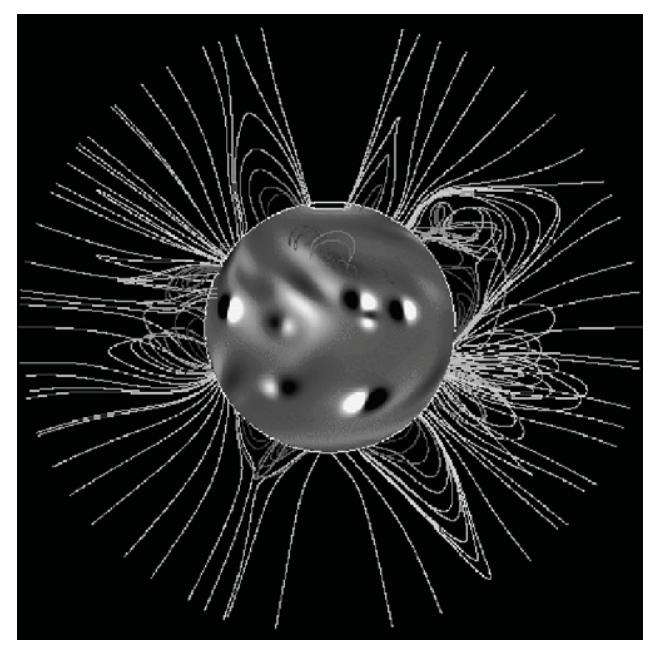

Figure 2. Example of the magnetic field distribution in the global simulation after 108 days of evolution, showing highly twisted flux ropes, weakly sheared arcades and near potential open fields. On the grey-scale image white/black represents positive/negative flux.

months to years (Yeates et al. 2007). Coupled to this is a quasi-static coronal evolution model, which uses a magnetofrictional relaxation technique (Yang et al. 1986) to evolve the coronal magnetic field through sequences of non-linear force-free fields in response to the observed photospheric evolution. In Figure 2 an example of the global field can be seen after 108 days of evolution where both the photospheric distribution (grey-scale image) and non-linear force-free coronal field are shown. The coronal field is made up of highly twisted flux ropes, slightly sheared coronal arcades and near potential open field lines. A key difference between this technique and previous studies, is that it can be run for extended periods without ever resetting the surface field back to that found in observations or the coronal field to potential. Therefore, the simulations are able to consider long-term helicity transport across the solar surface from low to high latitudes. Three applications of this model are now discussed.

\subsection{Hemispheric pattern of solar filaments}

Solar Filaments form over a wide range of latitudes on the Sun. They denote locations of highly non-potential magnetic fields and therefore locations of free magnetic energy and helicity storage. This helicity storage is seen through a hemispheric pattern that is observed in the direction of the axial magnetic field threading through filaments. The majority of filaments are found to be dextral/sinistral in the northern/southern hemipsphere (Martin et al. 1994). To test if the global code has the correct physics to explain the distribution and build-up of helicity in filaments, Yeates et al. (2008) carried out a one-to-one comparison between the observed chirality of 109 filaments and the chirality produced by the global model. The comparison covered a 6 month period and compared the observations and simulations at the exact time and location where the filaments were observed.

Through varying the sign and amount of helicity emerging within the bipoles, Yeates et al. (2008) (see their Figure 5b) show that by emerging dominantly negative helicity in the northern hemisphere and positive in the southern, a $96 \%$ agreement can be found between the observations and simulations, where the agreement is equally good for mi- 


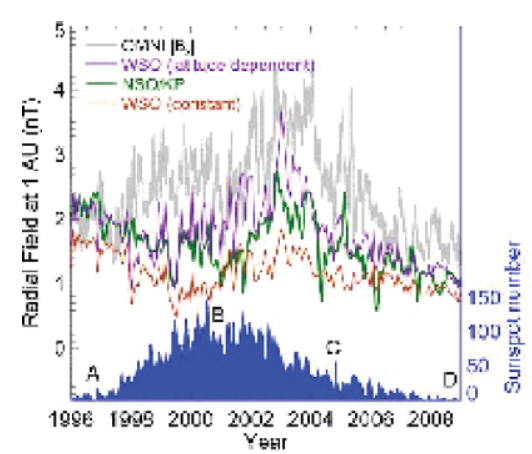

(a)

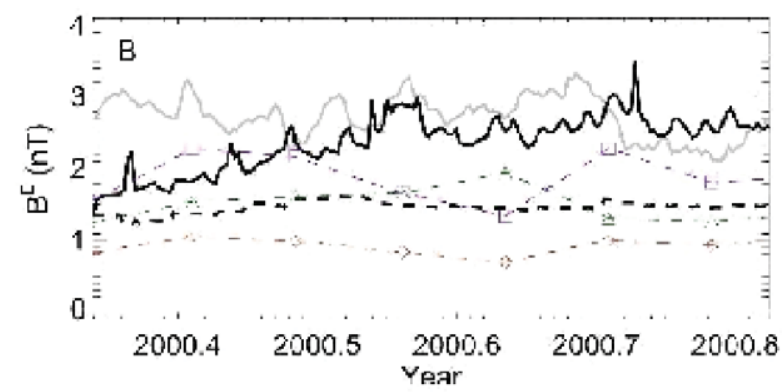

(b)

Figure 3. (a) Graph comparing open flux estimates from PFSS models to IMF field measurements (grey line). (b) Open flux estimate from non-potential simulations (black line) along with PFSS estimates (dashed) and IMF field measurements (grey line).

nority chirality filaments as well as for dominant chirality filaments. A key feature of the simulations is that a better agreement between the observations and simulations is found the longer the simulations are run. This indicates that the Sun has a long term memory of the transport of helicity from low to high latitudes. The reason for this high agreement is described in the paper of Yeates \& Mackay (2009).

\subsection{Open flux}

The Sun's Open Magnetic Flux is part of its magnetic field that extents out into interplanetary space and forms the Interplanetary Magnetic Field (IMF). However, measurements of the IMF over several solar cycles do not agree with open flux values deduced from PFSS models. In general PFSS models underestimate the open flux, where this is particularly apparent around cycle maximum. This is clearly shown in Figure 3(a) which compares various PFSS extrapolations with the IMF field. Through simulating the global corona over 4 distinct, 6-month periods (labelled A-D in Figure 3(a)), Yeates et al. (2010), showed that this discrepancy may be resolved through allowing electric currents to form in the global corona. This is illustrated in Figure 3(b) which simulates period B. The dashed lines denote open flux from various PFSS extrapolations, the grey line the observed IMF field strength and the black solid line the open flux from the global simulation. This clearly gives a much better agreement in absolute magnitude terms. Yeates et al. (2010) deduced that to produce this agreement, the open flux has three main contributions. The first is a background level due to the location of the flux sources (this component is also present in potential field models). The second is an enhancement due to electric currents which results in an inflation of the magnetic field. This inflation can be seen as the steady increase of the curve over the first rotation to a higher base level. Finally, there is a sporadic component to the open flux as a result of flux rope ejections. While this is one explanation for the shortfall other explanations have been put forward by a variety of authors (see Schüssler \& Baumann 2006; Riley 2007; Lockwood et al. 2009; Fisk \& Zurbuchen 2006)

\subsection{Magnetic flux ropes and CMEs}

Over the years a wide variety of mechanisms have been proposed for the initiation of CMEs (Forbes et al. 2006). One such mechanism is the flux rope ejection model (Lin 


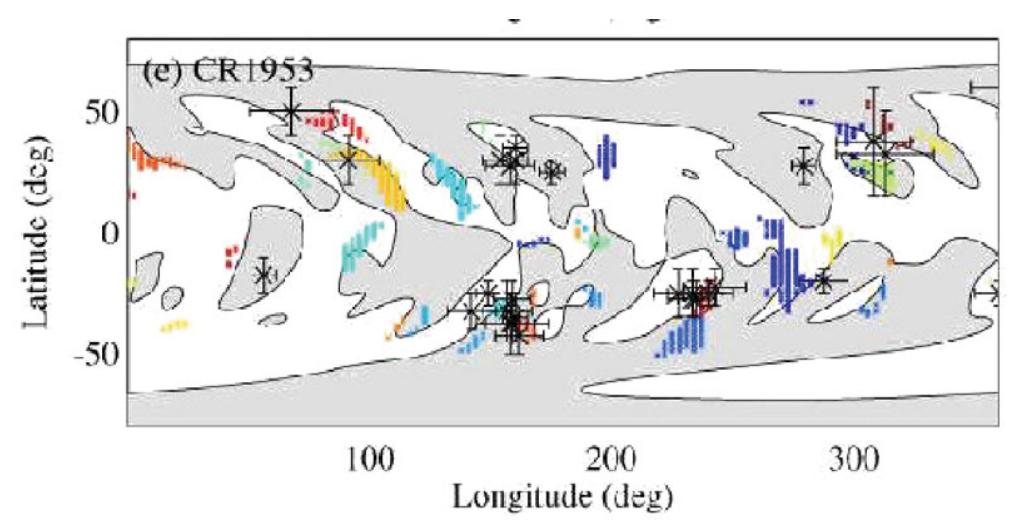

Figure 4. Comparison of CME source location from EUV images (crosses) with sites of flux rope ejections from the global non-potential simulations of Yeates et al. (2010) for CR1953.

et al. 2003) where surface shearing motions and flux cancellation produce a twisted structure which then loses equilibrium (van Ballegooijen \& Martens 1989). Within the global simulations of Yeates \& Mackay (2009) the formation of flux ropes is a natural consequence as flux cancels and is advected from low to high latitudes. As these flux ropes become larger, local losses of equilibrium may occur (Mackay \& van Ballegooijen 2006). In the paper of Yeates et al. (2010) local losses of equilibrium were compared to the observed sites of CMEs. First, over a 4.5 month period (May-Sept 1999) 330 CMEs were identified from Lasco observations. However, from the corona-graphic images the initiation sites in the low corona could not be identified. To determine these, the CMEs were cross-correlated with EUV features in EIT 195 Åmages. Of the 330 CMEs, only 98 could be clearly associated with low coronal signatures in the EUV images. This illustrates a problem in identifying CME initiation sites.

When these 98 events where cross correlated with the sites of flux rope ejections from the global model (Figure 4), agreement could be found in some but not all cases. Overall the best correlation between the model and CMEs was 0.49. The authors however identified two separate classes of CME. The first are those which do identify with flux rope ejection locations in the simulation. These account for $1 / 2$ and are the ones that produce the positive correlation. The other half were those located within the centres of active regions and frequently re-occur over short time scales. The global model was unsuccessful in re-producing these as it does not consider the internal structure or dynamics of active regions. While the comparison was only partially successful, it shows that flux rope formation and loss of equilibrium is an important model for CME initiation.

\section{Global MHD models}

In recent years significant advance has been made in the construction of realistic 3D global MHD models (Lionello et al. 2009; Downs et al. 2010). These models solve the resistive MHD equations, including a realistic energy equation (thermal conduction, radiative losses and coronal heating) along with modelling the upper chromosphere and transition region. Due to computational requirements, at present, these models are restricted to static photospheric boundary conditions and steady state coronal solutions. Through computing synthetic emission profiles and comparing these to 
the pass bands of 171, 195, 284 Ain EUV EIT images and X-ray images, the authors have shown that they may reproduce multi-spectral observations of the solar corona. The key element in doing this is the form of coronal heating used. In addition such models have reproduced the white light coronal emission seen during eclipse events (Rušin et al. 2010).

\section{References}

Antiochos, S. K., DeVore, C. R., \& Klimchuk, J. A. 1999, Astrophys. J., 510, 485

Balogh, A., Smith, E. J., Tsurutani, B. T., Southwood, D. J., Forsyth, R. J., \& Horbury, T. S. 1995, Science, 268, 1007

Barnes, G. 2007, Astrophys. J. Lett., 670, L53

Baumann, I., Schmitt, D., \& Schüssler, M. 2006, Astron. Astrophys, 446, 307

Cook, G. R., Mackay, D. H., \& Nandy, D. 2009, Astrophys. J., 704, 1021

\& Cremades, H., St. Cyr, O. C. 2007, Advances in Space Research, 40, 1042

Downs, C., Roussev, I. I., van der Holst, B., Lugaz, N., Sokolov, I. V., \& and Gombosi, T. I. 2010, Astrophys. J., 712, 1219

Fisk, L. A. \& Zurbuchen, T. H. 2006, Journal of Geophysical Research (Space Physics), 111, 9115

Forbes, T. G., et al. 2006, Space Science Reviews, 123, 251

Hathaway, D. H. 2010, Living Reviews in Solar Physics, 7, 1

Lin, J., Soon, W., \& Baliunas, S. L., 2003, New Astronomy Review, 47, 53

Lionello, R., Linker, J. A., \& Mikić, Z. 2009, Astrophys. J., 690, 902

Lockwood, M., Stamper, R., \& Wild, M. N. 1999, Nature, 399, 437

Lockwood, M., Rouillard, A. P., \& Finch, I. D. 2009, Astrophys. J., 700, 937

Mackay, D. H., Jardine, M., Cameron, A. C., Donati, J.-F., \& Hussain, G. A. J. 2004, Mon. Not. Roy. Astron. Soc., 354, 737

Mackay, D. H. \& van Ballegooijen, A. A. 2006, Astrophys. J., 641, 577

Mackay, D. H., Karpen, J. T., Ballester, J. L., Schmieder, B., \& Aulanier, G. 2010, Space Science Reviews, 151, 333

Martin, S. F., Bilimoria, R., \& Tracadas, P. W. 1994, Solar Surface Magnetism, 303

Riley, P. 2007, Astrophys. J. Lett., 667, L97

Rušin, V., et al. 2010, Astron. Astrophys, 513, A45

Schatten, K. H., Wilcox, J. M., \& Ness, N. F. 1969, Solar Phys., 6, 442

Schrijver, C. J. 2001, Astrophys. J., 547, 475

Schrijver, C. J. \& Title, A. M. 2001, Astrophys. J., 551, 1099

Schüssler, M. \& Baumann, I. 2006, Astron. Astrophys, 459, 945

Sheeley, N. R., Jr. 2005, Living Reviews in Solar Physics, 2, 5

Tandberg-Hanssen, E. 1995, Astrophysics and Space Science Library, 199, van Ballegooijen, A. A. \& Martens, P. C. H. 1989, Astrophys. J., 343, 971

van Ballegooijen, A. A., Priest, E. R., \& Mackay, D. H. 2000, Astrophys. J., 539, 983

Yang, W. H., Sturrock, P. A., \& Antiochos, S. K. 1986, Astrophys. J., 309, 383

Yeates, A. R., Mackay, D. H., \& van Ballegooijen, A. A. 2007, Solar Phys., 245, 87

Yeates, A. R., Mackay, D. H., \& van Ballegooijen, A. A. 2008, Solar Phys., 247, 103

Yeates, A. R. \& Mackay, D. H. 2009, Solar Phys., 254, 77

Yeates, A. R. \& Mackay, D. H. 2009, Astrophys. J., 699, 1024

Yeates, A. R., Attrill, G. D. R., Nandy, D., Mackay, D. H., Martens, P. C. H., \& van Ballegooijen, A. A. 2010, Astrophys. J., 709, 1238

Yeates, A. R., Mackay, D. H., van Ballegooijen, A. A., \& Constable, J. A. 2010, arXiv:1006.4011

Wang, Y.-M., Nash, A. G., \& Sheeley, N. R., Jr. 1989, Science, 245, 712 


\section{Discussion}

Manalis K. Georganlis: How do you ensure that you have a unique solution for the global solar field?

MACKAY: With - yeah, yeah, well, first of all, the distinction - what to make here is the type of model you are talking about is an extrapolation model where you specify a photospheric boundary condition which is the normal field component and essentially the vertical component of electric current, and then you can't produce a unique connectivity. Here we preserve our connectivity throughout the simulation mostly within it. There are some regions where, of course, we don't do that where there is strong reconnection sites. But in general the connectivity is preserved.

So when we start off with the connectivity of a bipolar merging, that connectivity will be preserved as its transferred across the surface. So we are forming a unique solution in terms of the initial connectivity start off, the new flux emergence, and the transport process. So if you run it again and again and again, you will get the same result. So it will always be the same result. So we don't have this uniqueness problem where you connect one field light to the next.

Kosovichev: A significant component of the Wang-Sheeley model is the process of diffusion of the leading magnitude flux across the equator. This is important for the polar field reversals. If the flux emerges only at high latitudes then it cannot diffuse to the equator, and then there will be no polarity reversals, which are important for the dynamo mechanism.

MACKEY: Well, we've - we've apply - we've only applied it to two stars, AB Duratus(spelled phonetically) and the other star, I can't remember. But Moira Jardine remembers the name, HD or something. What we find is, yes, we do for those two stars always need enhanced meridianal flow rates much larger than what occurs on the sun; but that does fit in with it being a rapid rotator.

Q.: So the only way you can get it faster is having a [INAUDIBLE]?

MACKEY: Yeah, well, yeah, I completely agree with you there. It's a tricky problem doing this. But remember the flux transport simulations are only representing the surface field. We don't take into account these other effects within them. So we do need to include more physics to really see what's going on. So this study was more initial speculative studies to see what was happening there.

But if we take the observed magnetograms to represent the surface field, that's what we find we need to include. Of course we have assumed many similar type solar parameters there. If the flux emergence is completely different, the profile is completely difference. Then of course you can get very different results, and we might need different surface transport parameters. So the more observations we get on the emergence latitudes of spots and the frequency and so on, the more we can be able to construe it.

Q.: How do you determine what type of helicity you inject?

MACKEY: That's as much as the question had previously there. At the moment we use statistical relationships, say, determined by Petsolve(spelled phonetically) et al. for a dominant sign of helicity in each hemisphere as the initial results because we can't 
constrain it any further than that. But with things like SDO and vector magnetic field measurements which will occur much more regularly, we can use them to constrain the helicity injection through new active regions. So that's the next stage in the models to make it much more realistic constraining it with observations. 